\title{
Safety and efficacy of endoscopic submucosal dissection for rectal neoplasia: a multicenter North American experience
}

\section{(ㄷ)(1) $\ominus$}

Authors

Dennis Yang ${ }^{1}$, Hiroyuki Aihara ${ }^{2}$, Yaseen B. Perbtani ${ }^{1}$, Andrew Y. Wang ${ }^{3}$, Abdul Aziz Aadam ${ }^{4}$, Yutaka Tomizawa ${ }^{5}$, Joo Ha Hwang ${ }^{5}$, Baiming Zou ${ }^{6}$, Nikola S. Natov ${ }^{3}$, Amanda Siegel ${ }^{4}$, Milad Pourmousavi Khoshknab ${ }^{7}$, Mouen A. Khashab ${ }^{7}$, Saowanee Ngamruengphong ${ }^{7}$, Harshit S. Khara ${ }^{8}$, David L. Diehl ${ }^{8}$, Thibaut Maniere ${ }^{9}$, Sherif Andrawes ${ }^{10}$, Petros Benias $^{10}$, Nikhil A. Kumta ${ }^{11}$, Fariha Ramay ${ }^{12}$, Raymond E. Kim ${ }^{12}$, Jason Samarasena ${ }^{13}$, Kenneth Chang ${ }^{13}$, Rintaro Hashimoto ${ }^{13}$, Benjamin Tharian ${ }^{14}$, Sumant Inamdar ${ }^{14}$, Gloria Lan ${ }^{15}$, Amrita Sethi ${ }^{15}$, Michael J. Nosler ${ }^{16}$, Abdalaziz Tabash $^{17}$, Mohamed O. Othman ${ }^{17}$, Peter V. Draganov ${ }^{1}$

\section{Institutions}

1 Division of Gastroenterology and Hepatology, University of Florida, Gainesville, FL, USA

2 Division of Gastroenterology and Hepatology, Brigham and Women's Hospital, Boston, MA, USA

3 Division of Gastroenterology and Hepatology, University of Virginia, Charlottesville, VA, USA

4 Division of Gastroenterology and Hepatology, Northwestern University Medical Center, Chicago, IL, USA

5 Division of Gastroenterology and Hepatology, University of Washington, Seattle, WA, USA

6 Department of Biostatistics, University of Florida, Gainesville, FL, USA

7 Division of Gastroenterology and Hepatology, Johns Hopkins Medicine, Baltimore, MD, USA

8 Division of Gastroenterology and Hepatology, Geisinger Health System, Danville, PA, USA

9 Division of Gastroenterology and Hepatology, Universite de Sherbrooke, Sherbrook, QC, Canada

10 Division of Gastroenterology and Hepatology, Northwell Health, Staten Island, NY, USA

11 Division of Gastroenterology, Mount Sinai Hospital, New York, NY, USA

12 Division of Gastroenterology and Hepatology, University of Maryland, Baltimore, MD, USA

13 Division of Gastroenterology and Hepatology, University of California Irvine, Irvine, CA, USA

14 Division of Gastroenterology and Hepatology, University of Arkansas Medical Sciences, Little Rock, AR, USA

15 Division of Gastroenterology and Hepatology, New York Presbyterian Hospital, New York, NY, USA

16 UC Health Medical Group, Fort Collins, CO, USA

17 Division of Gastroenterology and Hepatology, Baylor University Medical Center, Houston, TX, USA submitted 9.6.2019

accepted after revision 16.8.2019

Bibliography

DOI https://doi.org/10.1055/a-1010-5663 |

Endoscopy International Open 2019; 07: E1714-E1722

(c) Georg Thieme Verlag KG Stuttgart · New York

elSSN 2196-9736

Corresponding author

Dennis Yang, MD, 1329 SW 16th Street, Room \#5252,

Gainesville, FL 32608, USA

Fax: +1-352-627-9002

dennis.yang@medicine.ufl.edu

\section{ABSTRACT}

Background and aims Rectal lesions traditionally represent the first lesions approached during endoscopic submucosal dissection (ESD) training in the West. We evaluated the safety and efficacy of rectal ESD in North America. Methods This is a multicenter retrospective analysis of rectal ESD between January 2010 and September 2018 in 15 centers. End points included: rates of en bloc resection, R0 resection, adverse events, comparison of pre- and postESD histology, and factors associated with failed resection. Results In total, 171 patients (median age 63 years; 56\% men) underwent rectal ESD (median size $43 \mathrm{~mm}$ ). En bloc resection was achieved in 141 cases $(82.5 \%$; $95 \% \mathrm{Cl} 76.8-$ 88.2 ), including 24 of 27 (88.9\%) with prior failed endoscopic mucosal resection (EMR). R0 resection rate was $74.9 \%$ (95\% Cl 68.4-81.4). Post-ESD bleeding and perforation occurred in $4(2.3 \%)$ and 7 (4.1\%), respectively. Covert submucosal invasive cancer (SMIC) was identified in $8.6 \%$ of post-ESD specimens. There was one case $(1 / 120 ; 0.8 \%)$ of recurrence at a median follow-up of 31 weeks; IQR: 19-76 weeks). Older age and higher body mass index (BMI) were predictors of failed $\mathrm{R} 0$ resection, whereas submucosal fi- 
brosis was associated with a higher likelihood of both failed en bloc and $\mathrm{R} 0$ resection.

Conclusion Rectal ESD in North America is safe and is associated with high en bloc and $\mathrm{R} 0$ resection rates. The presence of submucosal fibrosis was the main predictor of failed en bloc and R0 resection. ESD can be considered for select rectal lesions, and serves not only to establish a definitive tissue diagnosis but also to provide curative resection for lesions with covert advanced disease.

\section{Introduction}

Endoscopic resection is the first-line treatment for mucosally confined colorectal neoplasia given its associated lower cost, morbidity, and mortality when compared to surgery [1,2]. Various endoscopic resection techniques have been introduced over the years, from conventional polypectomy to endoscopic mucosal resection (EMR) and, more recently, endoscopic submucosal dissection (ESD). The European Society of Gastrointestinal Endoscopy (ESGE) and the Japan Gastroenterological Endoscopy Society (JGES) recommend ESD for colorectal lesions with suspected superficial submucosal invasion (SMI) or for those that cannot be optimally removed en bloc by EMR [3, 4]. Furthermore, a recent American Gastroenterological Association (AGA) Clinical Practice Update has also endorsed ESD for select colorectal lesions, including those at increased risk for covert SMI [5].

However, the adoption of colorectal ESD in the USA and Canada has been limited due to its steep learning curve, higher risk profile when compared to EMR, and differences in training opportunities compared with Asia [6, 7]. Since early gastric cancer is rare in North America, rectal lesions have commonly become the de facto first lesions approached during ESD training, given the perceived lower technical difficulty of ESD in the rectum when compared to other anatomical sites in the colon [6]. However, data from North America on clinical outcomes associated with rectal ESD are lacking. This may be of particular importance as a recent meta-analysis and systematic review indicated a lower complete (R0) resection rate and higher risk for postoperative bleeding after ESD in the rectum [8]. The aim of this study was to evaluate the safety and efficacy of ESD for rectal neoplasia across various centers in North America.

\section{Methods}

\section{Study population}

This is a retrospective multicenter cohort study of consecutive patients $\geq 18$ years of age undergoing rectal ESD at 15 centers in the USA and Canada between January 2010 and September 2018. All patients provided informed consent for the procedures. This study was approved by the institutional review board for human research at each participating institution, with the University of Florida serving as the central coordinating center.

Rectal lesions were defined as any lesion with an upper margin located within $18 \mathrm{~cm}$ of the anal verge and/or when more than $50 \%$ of the lesion was situated within $15 \mathrm{~cm}$ from the anal verge. Indications for ESD included nongranular-type laterally spreading tumors (LST-NGs) of $\geq 20 \mathrm{~mm}$, granular-type (LSTGs) or mixed LST of $\geq 20-30 \mathrm{~mm}$, and lesions difficult to resect with conventional EMR (i.e. lesions located near or at the dentate line, those with non-lifting sign, prior failed EMR) $[3,4]$. We excluded neuroendocrine tumors, gastrointestinal stromal tumors, and patients with underlying inflammatory bowel disease and adenomatous polyposis. Data was collected from prospectively maintained institutional endoscopic reporting databases and by retrospective review of electronic medical records. The data obtained from all participating centers was then compiled into a central database.

\section{ESD procedure}

Cases were performed with i. v. conscious sedation, deep sedation or general anesthesia with endotracheal intubation, at the discretion of the endoscopist and anesthesiologist (if involved). Carbon dioxide was used for insufflation in all cases. With slight variations, rectal ESD was performed as previously described [3] ( $\triangleright$ Fig.1). Briefly, the procedures were performed with either a single-channel gastroscope (GIF HQ190; Olympus America, Inc., Center Valley, PA, United States) or colonoscope (CF H190Al or PCF H190L; Olympus America). Lesions were examined under high definition white light, near focus, and digital or dye-based chromoendoscopy. Lesions were described according to the Paris classification [9], and based on surface topography (granular or non-granular lateral spreading tumors) [10]. At the discretion of the endoscopist, the ESD knife tip was used to demarcate the outer margin for the resection area, approximately $5 \mathrm{~mm}$ from the lesion. The submucosal space was then expanded by injection of a lifting solution containing Methylene Blue or Indigo Carmine admixed with normal saline or with a viscous agent. The degree of submucosal fibrosis was determined based on the findings identified at the time of ESD and classified as F0 (no fibrosis), F1 (mild fibrosis), or F2 (severe fibrosis) [11]. Rectal ESD was then achieved by repeated cycles of injection and dissection with the electrocautery knife. Cases of failed rectal ESD were treated with either conventional EMR, hybrid ESD as defined by the JGES [3], or referred to surgery. Upon completion of the ESD, endoscopic closure of the resection bed using clips and/or endoscopic suturing was performed at the discretion of the endoscopist.

\section{Histopathology evaluation}

All ESD resected tissue specimens were pinned down with needles onto cork after removal and fixed using $10 \%$ formalin solution. All specimens were stained with hematoxylin and eosin. Histologic assessment was performed by pathologists from each respective participating center according to the World 

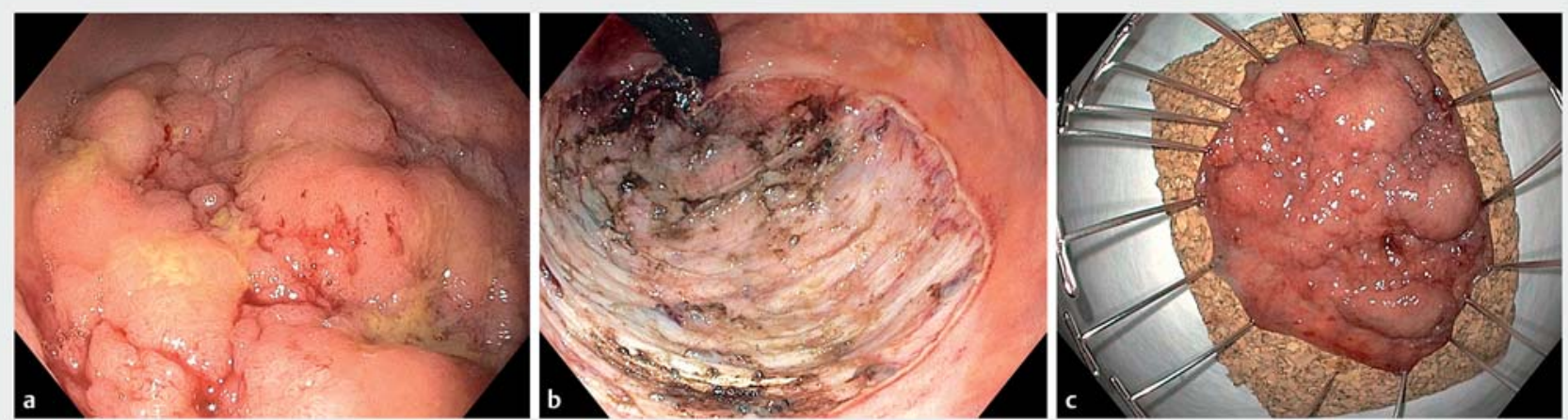

- Fig. 1 Rectal ESD procedure. a A $35 \mathrm{~mm}$ lesion (Paris classification 0-Ila + IIc) is identified in the rectum. b Post-ESD resection bed involving nearly $50 \%$ of the circumference. c ESD en bloc resected specimen.

Health Organization classification of colorectal neoplasia and the Vienna classification $[12,13]$.

\section{ESD operator experience}

All ESD procedures were performed by interventional endoscopists from each participating center. All of the endoscopists had long-standing experience in colorectal EMR, but varied in their experience with ESD. Colon ESD experience was defined as the total number of human colorectal ESD cases that each endoscopist had performed by the time of study enrollment. Novice and experienced colon ESD endoscopists were defined as those with fewer or more than 10 colorectal ESDs by the time of study participation, respectively.

\section{Outcome measures and definitions}

En bloc resection was defined as excision of the targeted lesion in a single specimen. Complete histologic resection (R0) was defined as resection with lateral and deep margins free of colorectal neoplasia. Incomplete histological resection was defined as failure to achieve neoplasia-free margins (R1), or when margins could not be adequately evaluated $(R x)$. Curative resection was defined when all of the following criteria were met: (1) resected specimen with negative lateral and deep margins of colorectal neoplasia, (2) depth of SMI $<1000 \mu \mathrm{m}$ below the muscularis mucosae, (3) absence of poorly differentiated or mucinous histology, and (4) absence of lymphovascular involvement and tumor budding $[3,4]$. A superficial invasive adenocarcinoma was defined as a lesion with SMI invasion $<1000 \mu \mathrm{m}$ below the muscularis mucosae. Recurrence referred to endoscopic and/or histological evidence of colorectal neoplasia at follow-up. Adverse events were defined based on previously established criteria by the American Society of Gastrointestinal Endoscopy (ASGE) [14].

\section{Statistical analysis}

Descriptive statistics for each baseline variable was obtained and expressed as mean and standard deviation (SD), or median and interquartile ranges (IQR). Chi-squared or Fisher exact test for categorical variables and the $t$ test for continuous variables were performed when indicated. Nominal $P$ values are report- ed; $P$ values less than 0.05 were considered significant. Multiple logistic regression analysis was performed to identify factors associated with failed en bloc, RO resection, and adverse events. Specifically, for each of the dependent variables, we conducted a backward model to select the optimal parsimonious model for the data. We started with a full model by including all of the observed baseline covariates and the least significant independent covariate was removed from the model until no more could be removed. All statistical analysis was performed with the open source statistical software package $R$ (version 3.5.0).

\section{Results}

\section{Baseline characteristics}

A total of 171 patients (median age 63, IQR: 55-72.5 years; $55.6 \%$ men) underwent rectal ESD across 15 centers in the United States $(n=14)$ and Canada $(n=1)$ between January 2010 and September 2018 ( $\triangleright$ Table 1 ). The median lesion size was $43 \mathrm{~mm}$ (IQR: 34.8-60 mm) and these were categorized according to the Paris classification ( $\triangleright$ Table 1 ). A total of 104 lesions $(60.8 \%)$ were classified as lateral spreading granular tumors (LST-G) and 38 (22.2\%) as non-granular lesions (LST-NG). Most of the lesions (142; $83 \%$ ) had previously been manipulated before ESD: 130 (76\%) were biopsied with forceps, 18 (10.5\%) had been tattooed, and 27 (15.8\%) had a prior EMR attempt. Rectal endoscopic ultrasound (EUS) was performed in 54/171 (31.6\%) before ESD. No pathological lymphadenopathy or invasion into the muscularis propria was reported in any of the EUS. Baseline histopathology (before ESD) was available in 151 out of 171 cases ( $\triangleright$ Table 1 ). Among these, nine patients with invasive adenocarcinoma (5.3\%) were deemed poor surgical candidates and thereby referred for ESD.

\section{Procedural characteristics}

Procedural characteristics are summarized in $>$ Table 2 . The median resected specimen size was $48 \mathrm{~mm}$ (IQR: $35-65 \mathrm{~mm}$ ) and located at a median of $4 \mathrm{~cm}$ (IQR: $1-8.3 \mathrm{~cm})$ to $9.5 \mathrm{~cm}$ (IQR: $6-14 \mathrm{~cm}$ ) from the anal verge. Twenty-nine (17\%) lesions were located at the dentate line. Submucosal fibrosis was en- 
- Table 1 Baseline characteristics.

Age, mean $\pm S D$, years

$63.4 \pm 11.7$

Sex, n (\%)

- Female

$76(44.4 \%)$

- Male

Body Mass Index; mean \pm SD, $\mathrm{kg} / \mathrm{m}^{2}$

$95(55.6 \%)$

$28.2 \pm 6.2$

ASA grade, $n(\%)$

. 1

- II

$23(13.5 \%)$

$82(48 \%)$

- III

$53(31 \%)$

- IV

$5(2.9 \%)$

- $N / A$

$10(5.8 \%)$

Lesion size, median (interquartile range), mm

$43(34.8-60)$

Gross morphology, n (\%)

- Lateral spreading granular tumor $104(60.8 \%)$

- Lateral spreading non-granular tumor $38(22.2 \%)$

- $N / A$

$27(15.8 \%)$

Paris Classification, n (\%)

\begin{tabular}{|c|c|}
\hline . Ip & $2(1.2 \%)$ \\
\hline - Is & $15(8.8 \%)$ \\
\hline - 0-lla & $45(26.3 \%)$ \\
\hline - $0-I I b$ & $7(4.1 \%)$ \\
\hline - $0-\| \mathrm{C}$ & $1(0.6 \%)$ \\
\hline - $0-I I I$ & 0 \\
\hline - IIa+IIc & $29(17 \%)$ \\
\hline - IIc+IIa & $2(1.2 \%)$ \\
\hline - IIa+ls & $33(19.3 \%)$ \\
\hline
\end{tabular}

Lesion manipulation before ESD, n (\%)

\section{- None}

$29(17 \%)$

- Tattoo at the lesion

- Cold biopsy forceps

$18(10.5 \%)$

$130(76)$

- Hot biopsy forceps

5 (2.9)

- Failed endoscopic mucosal resection (EMR)

- Endoscopic ablation

$27(15.8)$

$4(2.3)$

Pre-ESD histopathology, n (\%)

- Adenoma with LGD

87 (50.9)

- Adenoma with HGD

$52(30.4)$

- Sessile serrated adenoma/polyp

$3(1.8)$

- Invasive adenocarcinoma

- Not available

$20(11.7)$

ASA, American Society of Anesthesiologists; SD, standard deviation; ESD, endoscopic submucosal dissection; LGD, low grade dysplasia; HGD, high grade dysplasia.

- Table 2 Procedural characteristics.

\section{Type of anesthesia}

- Conscious sedation

$37(21.6 \%)$

- Monitored anesthesia care

$92(53.8 \%)$

- General anesthesia

$42(24.6 \%)$

Size of resected specimen; median (interquartile

$48(35-65)$

range), $\mathrm{mm}$

Lesion location; median (interquartile range), mm

- Distance of distal lesion margin from dentate line

$4(1-8.3)$

- Distance of proximal lesion margin from dentate

$9.5(6-14)$ line

Degree of submucosal fibrosis, n (\%)

- F0 (none)

- F1 (mild)

$43(25.1 \%)$

- F2 (severe)

$52(30.4 \%)$

Total procedure time; median (interquartile

$120(80-176)$ range), $\min$

Type of ESD knife used, $\mathrm{n}$ (\%)

- Dual knife

$116(67.8 \%)$

- Hybrid knife

$56(32.7 \%)$

- IT-knife

$45(26.3 \%)$

- Combination of ESD knives

$43(25.1)$

Epinephrine added to submucosal injection, n (\%)

- Yes

$77(45 \%)$

- No $94(55 \%)$

Elective endoscopic closure of post-ESD site, $n$ (\%)

- Yes

- No $113(66.1 \%)$

Hospitalization following ESD, n (\%)

- Yes

- No $97(56.7 \%)$

ESD histopathology, n (\%)

- Adenoma with LGD $85(49.7 \%)$

- Adenoma with HGD $63(36.8 \%)$

- Sessile serrated adenoma/polyp $3(1.8 \%)$

- Invasive adenocarcinoma $20(11.7 \%)$

ESD, endoscopic submucosal dissection; LGD, low grade dysplasia; HGD, high grade dysplasia. 
countered in most lesions (95; 55.6\%), of which 43 (25.1\%) and $52(30.4 \%)$ were categorized as mild (F1) and severe (F2), respectively. The median procedure time was 120 minutes (IQR: 80-176 minutes). Overall, the Dual Knife (Olympus America) was the most commonly used electrocautery ESD knife (116; $67.8 \%)$, followed by the Hybrid Knife (ERBE USA, Marietta, GA, United States) $(56 ; 32.7 \%)$ and IT-nano knife (Olympus America) $(45 ; 26.3 \%)$. Elective closure of the ESD resection site was performed in 58 of 171 cases (33.9\%) using endoscopic clips $(26 ; 15.2 \%)$, suturing $(27 ; 15.8 \%)$ or a combination thereof $(5 ; 2.9 \%)$. Most cases $(97 / 171 ; 56.7 \%)$ were performed in the outpatient setting. The mean post-procedural hospitalization stay was $0.5 \pm 0.7$ days. Final histopathology of the ESD resected specimens is shown in $>$ Table 2.

\section{Resection outcomes and adverse events}

Overall en bloc resection was achieved in 141 out of 171 cases (82.5\%; $95 \% \mathrm{Cl} 76.8-88.2)$, in 24 out of 27 rectal lesions (88.9\%) with previously failed EMR, and in $93.1 \%(27 / 29)$ of those located at the dentate line. En bloc resection rate was lower $(72.2 \%)$ in those with a tattoo at the lesion (13/18). Hybrid ESD $(23 / 30 ; 76.7 \%)$ or piecemeal EMR (7/30; $23.3 \%)$ was performed when conventional ESD failed. R0 (complete) resection was achieved in 128 of 171 cases $(74.9 \%$; $95 \% \mathrm{Cl} 68.4-$ 81.4). R1 resections were due to positive lateral margins ( $n=$ $13)$, deep margins $(n=4)$, both $(n=3)$, or uncertain $(R x)$ margins $(n=23)$. The overall curative resection rate was $73.1 \%$ (95\% Cl 66.5-79.8).

There were 20 patients with invasive adenocarcinoma on final pathology. Curative resection was achieved in 9 out of 11 cases $(81.8 \%)$ of superficially invasive adenocarcinoma, with the two non-curative resection cases due to positive lateral margins. Of these, one patient underwent surgery with no residual disease whereas the other one declined treatment. ESD was non-curative for the remaining nine cases of invasive adenocarcinoma due to: poorly differentiated histology $(n=3)$, lymphovascular involvement $(n=4)$, and/or submucosal invasion $>1000 \mu m(n=3)$. Two of these patients underwent surgery with no residual tumor or lymph node metastases in the resected specimens. The remaining seven patients were either unfit or declined additional treatment.

In all, there were 20 adverse events (11.7\%; $95 \% \mathrm{Cl} 7.7-17.4$ ) reported $(\triangleright$ Table 3$)$. There were three cases $(1.8 \%)$ of early bleeding ( $<24$ hours after ESD) and one case $(0.6 \%)$ of delayed bleeding on postoperative day \#5 upon antithrombotic therapy resumption. All cases of bleeding responded to endoscopic therapy. Perforation occurred in seven cases (4.1\%): 4 were closed endoscopically, 2 were managed expectantly with full recovery and one patient was referred for full-thickness transanal resection. The patient who underwent full-thickness transanal resection had invasive adenocarcinoma on baseline histopathology and had elected ESD over surgery based on personal preference and comorbidities. The perforation was deemed difficult to repair endoscopically, and upon discussion with colorectal surgery, the decision was to proceed with full-thickness transanal resection. Five patients $(2.9 \%)$ reported nausea and/ or abdominal pain, all adequately managed with anti-emetics
Table 3 Resection outcomes of rectal ESD.

Overall en bloc resection rate, $\mathrm{n}(\%)$

$141(82.5 \%)$

En bloc resection rate in complex lesions, $\mathrm{n}(\%)$

- Previously failed EMR ( $n=27)$

$24(88.9 \%)$

- Lesions at the dentate line $(n=29)$

$27(93.1 \%)$

- Tattoo at the lesion $(n=18)$

$13(72.2 \%)$

R0 resection, $\mathrm{n}(\%)$

$128(74.9 \%)$

$\mathrm{R} 1$ resection, $\mathrm{n}(\%)$

$20(11.7 \%)$

Rx resection, n (\%)

$23(13.5 \%)$

Overall curative resection, $\mathrm{n}(\%)$

$125(73.1 \%)$

Curative resection for superficial invasive adenocarcinoma $(n=11), n(\%)$

$9(81.8 \%)$

Adverse events, n (\%)

- Bleeding $4(2.3 \%)$

- Perforation $7(4.1 \%)$

- Nausea/abdominal pain $3(2.9 \%)$

- Postoperative urinary retention $3(1.8 \%)$

- Post-ESD anal stricture $1(0.6 \%)$

EMR, endoscopic mucosal resection; ESD, endoscopic submucosal dissection.

and analgesics. Urinary retention in three $(1.8 \%)$ patients resolved with short-term catheterization. An anal stricture developed in one patient after ESD of a nearly circumferential $70 \mathrm{~mm}$ adenoma with high grade dysplasia located $4 \mathrm{~cm}$ from the dentate line. The stricture responded to one session of endoscopic dilation.

\section{Comparison of pre- and post-ESD histopathology: covert invasive adenocarcinoma}

Tissue diagnosis was obtained for baseline histopathology in 151 of the 171 cases referred for ESD. Overall, the pre- and post-ESD histology matched in $80.1 \%(121 / 151)$ and was upstaged in $23(15.2 \%)$ of the 151 cases. Ten adenomas (9.2\%) with low grade dysplasia were upstaged to high grade dysplasia. Similarly, 13 (8.6\%) dysplastic lesions (11 HGD and 2 LGD) were upstaged to invasive adenocarcinomas on final histopathology. Conversely, seven lesions (4.6\%) were downstaged from invasive adenocarcinoma to high grade dysplasia $(n=2)$, and from high grade to low grade dysplasia $(n=5)$.

\section{Follow-up}

A total of 120 out of the 171 patients (70.2\%) underwent surveillance endoscopy at a median of 23 weeks (IQR: 12-24 weeks) after ESD. None of the patients had undergone surgery at the time of surveillance endoscopy. The remaining 51 patients $(29.8 \%)$ were lost to follow-up. There was one case (1/120; $0.8 \%)$ of recurrence on follow-up (median 31 weeks; IQR: 19-76 weeks). This patient had initial curative resection for a well-differentiated adenocarcinoma with superficial SMI 
and no lymphovascular involvement. Repeat biopsies on surveillance confirmed invasive adenocarcinoma. Further treatment was not pursued given the patient's advanced age and comorbidities.

\section{Endoscopist experience}

The procedures were performed by endoscopists with varied colon ESD experience ( $\triangleright$ Table 4). The overall endoscopists' median colon ESD experience was 1 (IQR: 0.5-3.5). Most (14/15) of the providers were considered novice ESD endoscopists $(<10$ colon ESDs) at the time of study participation. One endoscopist (study center \#1) was highly experienced in colon ESD, having previously performed 350 colorectal ESDs. The en bloc resection and adverse event rates for each participating center are shown in $>$ Table 4 . There was no statistically significant difference between the overall en bloc resection rate and that among novice endoscopists ( $82.5 \%$; $95 \% \mathrm{Cl} 76.8-88.2$ vs. $77.1 \%$; $95 \% \mathrm{Cl}$ $68.8-83.8 ; P=0.31)$ nor in the adverse event rates $(11.7 \% ; 95 \%$ Cl $7.7-17.4$ vs. $14.3 \%$; $95 \% \mathrm{Cl} 8.3-23.2 ; P=0.57)$.

\section{Predictors of treatment outcomes and adverse events}

A multivariate logistic regression was performed to identify predictors of failed en bloc and $\mathrm{R} 0$ resection ( $\downarrow$ Table 5 ). We included age, gender, body mass index (BMI), American Society of Anesthesiologists (ASA) grade, lesion size, morphology (LST-G vs LST-NG), location (distance from the dentate line), prior EMR attempted (yes vs. no), presence of submucosal fibrosis, and endoscopist colorectal ESD experience as covariates.

Mild (F1) submucosal fibrosis was a predictor for en bloc resection failure (OR 30.65; $95 \% \mathrm{Cl} 2.244-1105 ; P=0.023$ ), whereas severe (F2) submucosal fibrosis was a strong predictor of both failed en bloc (OR: 166.5; $95 \% \mathrm{Cl} 12.21-8719 ; P=0.002$ ) and R0 resection (OR: 21.86; $95 \% \mathrm{Cl} 2.466-3365 ; P=0.012$ ). Both increasing age and BMI were associated with significantly higher odds of R0 resection failure ( $\downarrow$ Table 5 ). Lesion size, morphology, location, prior EMR attempts, and endoscopist experience were not predictive of en bloc or R0 resection failure. There were no significant factors predictive of adverse events ( Table 5).

\section{Discussion}

This is the first multicenter study from North America reporting clinical outcomes of ESD for rectal neoplasia. Although EMR remains the first-line treatment for most non-malignant colorectal lesions in North America, ESD has gain acceptance for select lesions, as supported by a recent AGA Clinical Practice Update [5]. The main limitation of EMR is the piecemeal nature of the resection when involving lesions larger than $20 \mathrm{~mm}$, which limits accurate histopathological evaluation and is associated with a higher recurrence rate [7,15-17]. In this study, en bloc and R0 resection with ESD was achieved in $82.5 \%$ and $74.9 \%$ of the lesions, respectively, which is comparable with data originating from Europe [8]. Furthermore, on follow-up at a median of 31 weeks, recurrence was detected in only one patient $(0.9 \%)$
- Table4 Endoscopist experience per center and ESD outcomes.

\begin{tabular}{|c|c|c|c|l|}
\hline $\begin{array}{l}\text { Study } \\
\text { center }\end{array}$ & $\begin{array}{l}\text { Colon } \\
\text { ESD ex- } \\
\text { perience }\end{array}$ & $\begin{array}{l}\text { \# Cases } \\
\text { in study }\end{array}$ & $\begin{array}{l}\text { En bloc } \\
\text { resection } \\
\text { rate, } \mathbf{n}(\%)\end{array}$ & $\begin{array}{l}\text { Adverse } \\
\text { event rate, } \\
\mathbf{n}(\%)\end{array}$ \\
\hline 1 & 350 & 40 & $40(100)$ & $2(5)$ \\
\hline 2 & 8 & 6 & $6(100)$ & $1(16.7)$ \\
\hline 3 & 2 & 4 & $1(25)$ & $1(25)$ \\
\hline 4 & 0 & 3 & $3(100)$ & $0(0)$ \\
\hline 5 & 0 & 33 & $29(87.9)$ & $4(12.1)$ \\
\hline 6 & 1 & 4 & $2(50)$ & $0(0)$ \\
\hline 7 & 1 & 9 & $6(66.7)$ & $1(11.1)$ \\
\hline 8 & 1 & 5 & $5(100)$ & $0(0)$ \\
\hline 9 & 1 & 10 & $5(50)$ & $1(10)$ \\
\hline 10 & 0 & 12 & $10(83.3)$ & $2(16.7)$ \\
\hline 11 & 5 & 8 & $5(62.5)$ & $0(0)$ \\
\hline 12 & 1 & 10 & $8(80)$ & $2(20)$ \\
\hline 13 & 0 & 8 & $5(62.5)$ & $2(25)$ \\
\hline 14 & 2 & 8 & $7(87.5)$ & $2(25)$ \\
\hline 15 & 5 & 11 & $9(81.8)$ & $2(18.2)$ \\
\hline ESD, endoscopic submucosal dissection. & & & \\
\hline & & & & \\
\hline
\end{tabular}

which is on par with the very low recurrence rate consistently demonstrated after ESD $[18,19]$.

EMR can be technically challenging in lesions with extensive submucosal fibrosis or in difficult locations [20,21]. Our data demonstrates that ESD was quite successful in difficult-to-treat rectal neoplasia. Most of the lesions (55.5\%) in this study were complicated by submucosal fibrosis, yet en bloc resection with ESD was still achieved in most of these difficult cases (70/95; $73.7 \%$ ). Furthermore, ESD also permitted en bloc resection of nearly all lesions located at the dentate line (93.1\%) or those with prior failed EMR (88.9\%). The efficacy of ESD in these challenging scenarios should not be understated, as failed endoscopic resection can incur (1) additional costs, (2) a potential risk of progression from under-recognition of residual/recurrent neoplasia following piecemeal resection, and (3) the burden of repeated invasive measures. In all, ESD can be considered to be a first-line or salvage therapy in difficult-to-resect rectal neoplasia, which is concordant with current Japanese and European guidelines, and the recent AGA Update [3-5].

Accurate pretreatment oncological staging is crucial in directing patient care. Nevertheless, despite our best efforts, neoplastic lesions in the rectum are often either under- or overstaged [22-27]. In our study, there were 13 cases (8.6\%) of covert invasive adenocarcinoma on final ESD histopathology. ESD was curative in $81.8 \%$ of invasive adenocarcinomas with superficial SMI and favorable histologic features. Conversely, piecemeal resection with EMR would have potentially subjected all of these patients to unwarranted surgery. Hence, en bloc resec- 
Table 5 Predictors of failed en bloc resection, failed R0 resection and adverse events.

\begin{tabular}{|c|c|c|c|}
\hline Variable & OR & $95 \% \mathrm{Cl}$ & $P$ value \\
\hline \multicolumn{4}{|l|}{ Failed en bloc resection } \\
\hline Age & 1.106 & $(0.992,1.232)$ & 0.069 \\
\hline Lesion size & 0.997 & $(0.949,1.043)$ & 0.903 \\
\hline Morphology (LST-G vs LST-NG) & 0.857 & $(0.112,7.566)$ & 0.881 \\
\hline At dentate line (yes vs. no) & 1.229 & $(0.965,1.718)$ & 0.139 \\
\hline Prior EMR attempt (yes vs no) & 4.469 & $(0.372,89.02)$ & 0.267 \\
\hline Colonic ESD experience & 1.025 & $(0.873,1.134)$ & 0.608 \\
\hline \multicolumn{4}{|l|}{ Submucosal fibrosis } \\
\hline - F1 & 30.65 & $(2.244,1105)$ & 0.023 \\
\hline . F2 & 166.5 & $(12.21,8719)$ & 0.002 \\
\hline \multicolumn{4}{|l|}{ Failed $\mathrm{R} 0$ resection } \\
\hline Age & 1.104 & $(1.021,1.216)$ & 0.023 \\
\hline BMI & 1.191 & $(1.042,1.414)$ & 0.022 \\
\hline \multicolumn{4}{|l|}{ ASA grade } \\
\hline .2 & 4.567 & $(0.226,257.5)$ & 0.370 \\
\hline .3 & 13.64 & $(0.486,1089)$ & 0.160 \\
\hline . 4 & 0.000 & $(0.000,2 e+59)$ & 0.993 \\
\hline Lesion size & 1.023 & $(0.975,1.073)$ & 0.332 \\
\hline Morphology (LST-G vs LST-NG) & 1.266 & $(0.206,9.103)$ & 0.802 \\
\hline At dentate line (yes vs no) & 1.170 & $(0.927,1.532)$ & 0.207 \\
\hline Prior EMR attempt (yes vs no) & 5.566 & $(0.435,90.23)$ & 0.190 \\
\hline Colonic ESD experience & 1.025 & $(0.953,1.119)$ & 0.536 \\
\hline \multicolumn{4}{|l|}{ Submucosal fibrosis } \\
\hline - F1 & 2.417 & $(0.268,22.44)$ & 0.421 \\
\hline - F2 & 21.86 & $(2.466,3.365)$ & 0.012 \\
\hline \multicolumn{4}{|l|}{ Adverse events } \\
\hline Age & 1.145 & $(0.996,1.391)$ & 0.096 \\
\hline Male & 2.585 & $(0.314,25.80)$ & 0.385 \\
\hline BMI & 1.036 & $(0.872,1.229)$ & 0.666 \\
\hline \multicolumn{4}{|l|}{ ASA grade } \\
\hline .2 & 1.055 & $(0.049,30.81)$ & 0.973 \\
\hline .3 & 0.310 & $(0.003,21.48)$ & 0.585 \\
\hline .4 & 0.000 & $(0.000,2 \mathrm{e}+258)$ & 0.998 \\
\hline Lesion size & 0.981 & $(0.921,1.039)$ & 0.510 \\
\hline Morphology (LST-G vs LST-NG) & 0.486 & $(0.050,4.003)$ & 0.501 \\
\hline At dentate line (yes vs no) & 0.691 & $(0.389,0.995)$ & 0.105 \\
\hline Prior EMR attempt (yes vs no) & 141.5 & $(0.540,37032)$ & 0.081 \\
\hline Colonic ESD experience & 1.048 & $(0.948,1.197)$ & 0.397 \\
\hline \multicolumn{4}{|l|}{ Submucosal fibrosis } \\
\hline - F1 & 0.000 & $(0.000,1.6 e+96)$ & 0.995 \\
\hline . F2 & 4.465 & $(0.261,136.6)$ & 0.321 \\
\hline
\end{tabular}

ASA, American Society of Anesthesiologists; BMI, Body Mass Index; EMR, endoscopic mucosal resection; ESD, endoscopic submucosal dissection. LST-G, laterally spreading tumor, granular type; LST-NG. laterally spreading tumor, non-granular type. 
tion is of particular importance for curative resection of lesions with suspected SMI, including large LST lesions with unsuspected advanced histopathology. And while ESD may be non-curative in some cases of invasive adenocarcinoma, prior data indicate that non-curative ESD does not compromise future attempts at surgery [25]. Similar to prior reports on gastric neoplasia, our study demonstrated that histologic discordance between forceps biopsy and ESD is not uncommon. Factors associated with downgrade compared to upgrade histology have included smaller tumor size and a lower number of biopsy specimens, further highlighting the limitations of establishing a diagnosis with forceps biopsies [28, 29]. Hence, when pretreatment diagnosis is uncertain or fails to identify advanced disease, ESD may prove to be an accurate staging procedure to guide subsequent management.

The transition to colorectal ESD in North America has been slower than its adoption in Asia and Europe, particularly due to the concern for serious adverse events. In our study, ESD was associated with an adequate safety profile, even though nearly all of our endoscopists had limited (less than 10) colon ESD experience before this study. It should be important to highlight that most cases of perforation $(5 / 7 ; 71.4 \%)$ occurred in lesions with submucosal fibrosis. Given that severe submucosal fibrosis was a predictor for both failed en bloc and R0 resection, we suggest that ESD trainees should avoid lesions with suspected submucosal fibrosis in the earlier stages of their training. Furthermore, increasing BMI was also associated with a significantly higher odds of R0 resection failure. Patients are often repositioned during ESD to optimize visualization during dissection and to maximize traction force with the use of gravity. We speculate that patient positioning may be more challenging and performed less frequently in obese patients, thereby accounting for the lower R0 resection rates in this subgroup. In this study, most ESD cases were performed in the outpatient setting, suggesting that hospitalization may not be routinely required, thereby limiting procedure-related costs. Given the steep learning curve, ESD of large complex colorectal polyps should be reserved to high-volume specialized centers. Future long-term prospective studies are needed to better define not only the role of ESD in the rectum but also for the treatment of lesions in the rest of the colon.

We acknowledge the limitations of this study. The study was retrospective and limited by its uncontrolled design and inherent selection bias. All ESD cases were performed by endoscopists trained in advanced endoscopic resection techniques; thus, results may not be generalizable. Furthermore, differences in ESD training background among the participating endoscopists may have led to performance variability. Nonetheless, with the exception of one provider, most of our endoscopists were novices in colon ESD and our results included all consecutive cases during this initial phase, thereby providing a more realistic and accurate picture on the current status of rectal ESD in North America.

We also recognize that, in the absence of central pathology review, discrepancies in diagnosis and staging of lesions between centers could have affected our results. Another significant limitation is that nearly one-third of the patients did not undergo surveillance (lost to follow-up) and the relatively short follow-up prohibits significant conclusions on long-term outcomes. Furthermore, in the absence of a pre-specified followup protocol, it is possible that delayed adverse events could have been missed and underestimated in our analysis. In addition, while this is one of the largest studies on rectal ESD, the relatively small sample size may have impeded finding statistically significant differences in outcomes among the three training phases. Lastly, the lack of comparison to alternative methods, such as piecemeal EMR, limits conclusions on the role of colorectal ESD.

In summary, our study demonstrates that rectal ESD in North America is safe and associated with high resection rates, even in difficult lesions. ESD should be considered for select rectal lesions, particularly those with possible SMI, as it can serve as an oncologic staging tool and is curative for superficially invasive covert advanced disease.

\section{Competing interests}

D Yang, PV Draganov, HS Khara, H Aihara, D Diehl, NA Kumta, AA Aadam, S Ngamruengphong, MA Khashab, A Sethi, J Samarasena, $\mathrm{K}$ Chang are consultants for Boston Scientific. $\mathrm{K}$ Chang, J Samarasena, A Sethi, MA Khashab, NA Kumta, H Aihara, PV Draganov, and $\mathrm{H}$ Khara are consultants for Olympus Inc. K Chang, J Samarasena, D Diehl, PV Draganov are consultants for Cook Medical. K Chang, J Samarasena, M Khashab, D Diehal, and $\mathrm{H}$ Khara are consultants for Medtronic. K Chang and NA Kumta are consultants for Apollo Endosurgery. $\mathrm{K}$ Chang and $\mathrm{H}$ Khara are consultants for Covidien. $\mathrm{K}$ Chang is consultant for ERBE, Endogastric solutions, Ovesco, Mederi and Torax. $\mathrm{H}$ Khara is consultant for ConMed and C2Therapeutics. K Chang and J Samarasena are consultants for Mauna Kea. K Chang, J Samarasena and D Diehl are consultants for Pentax. A Sethi and D Yang are consultants for US Endoscopy. D Diehl, H Aihara, D Yang and PV Draganov are consultants for Lumendi. H Aihara is consultant for Merit Endotek, GI Supply, Fujifilm Medical and Auris Health. J Samarasena is owner of Docbot Inc. PV Draganov is consultant for Microtech.

\section{References}

[1] Jayanna M, Burgess NG, Sing R et al. Cost analysis of endoscopic mucosal resection vs surgery for large laterally spreading colorectal lesions. Clin Gastroenterol Hepatol 2016; 14: 271-278

[2] Ahlenstiel G, Hourigan LF, Brown G et al. Actual endoscopic versus predicted surgical mortality for treatment of advanced mucosal neoplasia of the colon. Gastrointest Endosc 2014; 80: 668-676

[3] Tanaka S, Kashida H, Saito Y et al. JGES guidelines for colorectal endoscopic submucosal dissection/endoscopic mucosal resection. Dig Endosc 2015; 27: 417-434

[4] Pimentel-Nunes P, Dinis-Ribeiro M, Ponchon T et al. Endoscopic submucosal dissection: European Society of Gastrointestinal Endoscopy (ESGE) Guideline. Endoscopy 2015; 47: 829-854 
[5] Draganov PV, Wang AY, Othman MO et al. Clinical practice of endoscopic submucosal dissection in the United States. Clin Gastroenterol Hepatol 2019; 17: 16-25

[6] Kotzev Al, Yang D, Draganov PV. How to master endoscopic submucosal dissection in the USA. Dig Endosc 2019; 31: 94-100

[7] Yang D, Othman M, Draganov PV. Endoscopic mucosal resection vs endoscopic submucosal dissection for Barrett's esophagus and colorectal neoplasia. Clin Gastroenterol Hepatol 2019; 17: 1019-1028

[8] Fuccio L, Hassan C, Ponchon T et al. Clinical outcomes after endoscopic submucosal dissection for colorectal neoplasia: a systematic review and meta-analysis. Gastrointest Endosc 2017; 86: 74-86

[9] The Paris endoscopic classification of superficial neoplastic lesions: esophagus, stomach, and colon: November 30 to December 1, 2002. Gastrointest Endosc 2003; 58: S3-S43

[10] Kudo S, Lamber R, Allen JI et al. Nonpolypoid neoplastic lesions of the colorectal mucosa. Gastrointest Endosc 2008; 68: S3-S47

[11] Kim EK, Han DS, Ro Y et al. The submucosal fibrosis: what does it mean for colorectal endoscopic submucosal dissection? Intest Res 2016; 14: 358-364

[12] Hamilton SR, Bosman FT, Boffetta P et al. Carcinoma of the colon and rectum. WHO classification of tumours of the digestive system. J Clin Ultrasound 2014; 1: 1-6

[13] Schlemper RJ, Riddel RH, Kato Y et al. The Vienna classification of gastrointestinal epithelial neoplasia. Gut 2000; 47: 251-255

[14] Cotton PB, Eisen GM, Aabakken L et al. A lexicon for endoscopic adverse events: report of an ASGE workshop. Gastrointest Endosc 2010; 71: 450-454

[15] Benson AB 3rd, Venook AP, Cederquist L et al. Colon cancer, Version 1. 2017, NCC Clinical Practice Guideline in Oncology. J Natl Compr Canc Netw 2017; 15: 370-398

[16] Oka S, Tanaka S, Saito Y et al. Local recurrence after endoscopic resection for large colorectal neoplasia: a multicenter prospective study in Japan. Am J Gastroenterol 2015; 110: 697-707

[17] Khashab M, Eid E, Rusche M et al. Incidence and predictors of "late" recurrences after endoscopic piecemeal resection of large sessile adenomas. Gastrointest Endosc 2009; 70: 344-349
[18] Russo P, Barbeiro S, Awadie H et al. Management of colorectal laterally spreading tumors: a systematic review and meta-analysis. Endosc Int Open 2019; 7: E239-E259

[19] Terasaki M, Tanaka S, Oka S et al. Clinical outcomes of endoscopic submucosal dissection and endoscopic mucosal resection for laterally spreading tumors larger than $20 \mathrm{~mm}$. J Gastroenterol Hepatol 2012; 27: $734-740$

[20] Pidala M], Cusick MV. The difficult colorectal polyp. Surg Clin North Am 2017; 97: 515-527

[21] Ngamruengphong S, Pohl H, Haito-Chavez Y et al. Update on difficult polypectomy techniques. Curr Gastroenterol Rep 2016; 18: 3

[22] Hassan C, Repici A, Sharma P et al. Efficacy and safety of endoscopic resection of large colorectal polyps: a systematic review and meta-analysis. Gut 2016; 65: 806-820

[23] Moss A, Bourke M], Williams S] et al. Endoscopic mucosal resection outcomes and prediction of submucosal cancer from advanced colonic mucosal neoplasia. Gastroenterology 2011; 140: 1909-1918

[24] Barendse R, Musters GD, de Graaf EJR et al. Randomised controlled trial of transanal endoscopic microsurgery versus endoscopic mucosal resection for large rectal adenomas (TREND Study). Gut 2017; 67: 837-846

[25] Bronzwaer M, Musters GD, Barendse R et al. The occurrence and characteristics of endoscopically unexpected malignant degeneration in large rectal adenomas. Gastrointest Endosc 2017; 87: 862871

[26] Yang D, Draganov PV. Removing large rectal polyps: when the whole may be greater than the sum of its parts. Gastrointest Endosc 2018; 87: 872-874

[27] Yang D, Draganov PV. Endoscopic submucosal dissection (ESD) in 2018: A minimally invasive curative approach for select rectal lesions. Am J Gastroenterol 2019; 114: 687

[28] Kim JH, Kim Y], An J et al. Endoscopic features suggesting gastric cancer in biopsy-proven gastric adenoma with high-grade neoplasia. World J Gastroenterol 2014; 20: 12233-12240

[29] Yang MJ, Shin S], Lim SG et al. Comparison of clinical and endoscopic characteristics among upgrade, concordance and downgrade pathology after ESD. Gastrointest Endosc 2014; 81: AB250 